\title{
The Potential of TPP Chitosan Nanoparticles as Carrier for Poorly soluble Rosiglitazone Maleate
}

\author{
Vandana Singh ${ }^{1 *}$, Dr. Amrendra Kumar Chaudhary ${ }^{2}$ \\ 1. Translam Institute of Pharmaceutical Education and Research, Meerut, India. \\ 2. Department of Pharmacy, L.L.R.M. Medical College, Meerut, India. \\ *Corresponding author's E-mail: vandankhushi@gmail.com
}

Received: 18-07-2020; Revised: 24-09-2020; Accepted: 03-10-2020; Published on: 20-10-2020.

\section{ABSTRACT}

The objective of this study is to develop biodegradable sub-micron chitosan nanoparticles loaded with rosiglitazone maleate for intravenous drug targeting. The rosiglitazone maleate loaded chitosan nanoparticles were prepared by ionic gelation of chitosan with tri polyphosphate anions (TPP). The effects of chitosan concentration and sodium tri polyphosphate on the physicochemical properties of the nanoparticles were studied. The particle size and zeta potential of nanoparticles were determined, respectively by transmission electron microscopy (TEM) and a zeta potential analyzer. It was found that the nanoparticles showed a size distribution in the range of $86 \mathrm{~nm}-110 \mathrm{~nm}$ with spherical morphology and smooth surface structure. The encapsulation efficiency decreased with the increase of chitosan concentration. These studies showed that chitosan can form complex with tri polyphosphate anions to form stable cationic nanoparticles for subsequent rosiglitazone maleate. In vitro release studies showed a burst effect at the beginning and then a sustained release characteristic for $32 \mathrm{~h}$. The study on the drug to polymer ratio showed a linear relationship between the concentration of drug and percentage drug loading.

Keywords: TPP, chitosan, rosiglitazone maleate, TEM.

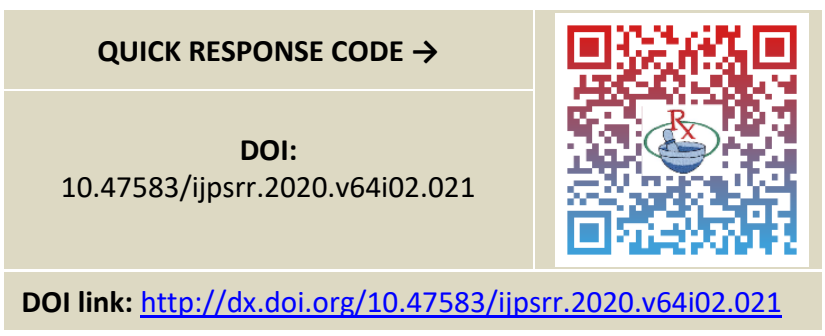

\section{INTRODUCTION}

$\mathrm{n}$ the recent past, substantial scientific and technological advancements have been made in the research and development of rate controlled oral drug delivery systems to counter the short comings of physiological adversities of conventional drugs and its administration. ${ }^{1}$ The rate controlled oral drug delivery system has given impetus to significant advancements in the pharmaceutical engineering of novel dosage forms such as nanoparticles, which are solid colloidal polymeric carriers than $1 \mu \mathrm{m}$ in size. ${ }^{2,3}$ These nanoparticles offers great advantages right from helping to increase the stability of drugs, proteins and up to controlled drug release properties. Several attempts have been made, towards developing biodegradable polymeric nanoparticles as potential drug delivery devices. In addition to the inherent property of reduced cytotoxicity, biodegradable polymeric nanoparticles have been found to be extremely effective in controlled and targeted drug release, and time-controlled drug delivery system. ${ }^{4}$

Diabetes mellitus is a vast growing public health problem throughout the world because of its association with increased cardio vascular mortality. So, the present exercise is focused towards anti-diabetic treatments.
Rosiglitazone maleate, a fast and short acting thiazolidinedione analog, is chosen as the model drug candidate for polymeric nanoparticles formulation. It has short half-life 3-4 $\mathrm{h}$ and has poor absorption characteristics in the upper intestinal tract. Although the drug is highly insulin sensitive, it suffers major drawbacks such as hepatic toxicity, anemia, G.I.T disturbances, oedema, liver toxicity and muscle cramps. ${ }^{5}$ Nano drug delivery system enclosed anti-diabetic drug which improve the therapeutic efficacy of the drug with pre-determined controlled, prolonged duration. Thus, the adverse effects, due to conventional dose can be surmounted. Recently many studies are focused on safety issues of manufactured nano materials to minimize or eliminate their nano toxicity before they are being widely used. ${ }^{6-9}$ Chitosan (CS) is the second abundant polysaccharide and a cationic polyelectrolyte present in nature. Chitosan is a polysaccharide comprising copolymers of glucosamine and $\mathrm{N}$-acetylglucosamine and can be derived by the partial deacetylation of chitin. It is a biodegradable, biocompatible and hydrophilic polymer of low toxicity. ${ }^{10}$ It is a material found in abundance in shells of crustacea such as lobsters, prawns and crabs. It is insoluble under alkaline and neutral conditions, but soluble with inorganic and organic acids such as hydrochloric acid, lactic acid, acetic acid and glutamic acid under acidic conditions. Therefore, the major goal of the present work is to create a new kind of biodegradable nanoparticles for the incorporation of rosiglitazone maleate and to evaluate their potential as delivery system. The factors that influence the preparation of nanoparticles were analyzed and their release property was examined. Hence in the present study we have made an attempt to 
optimize and to check the suitability and potentiality of natural carrier such as chitosan for diabetic drugs.

\section{MATERIALS AND METHODS}

Rosiglitazone maleate was obtained as a gift sample by Torrent Pharmaceuticals Ltd, Ahmedabad and chitosan was procured as a gift sample from Central Institute of Fisheries Technology (degree of deacetylation 85\%). Sodium tri polyphosphate was purchased by Qualikens fine Pvt Ltd., New Delhi and Tween 80 LR was purchased by S.D. Fine Chemicals Ltd, Mumbai. Dialysis membrane was purchased from Himedia laboratories (P) Ltd., Mumbai. All other reagents used were of analytical grade.

\section{Development of polymeric nanoparticles}

Chitosan nanoparticles were prepared by ionic gelation method. ${ }^{11}$ Chitosan was dissolved in acetic aqueous solution at various concentrations $(1.0,1.2,1.44,1.6,2.0$, $2.5,3.0 \mathrm{mg} / \mathrm{ml}$ ). The concentration of acetic acid in aqueous solution was, in all case 1.5 times that of chitosan. Under magnetic stirring, at room temperature, $4 \mathrm{ml}$ sodium tri polyphosphate aqueous solution with various concentrations $(0.2,0.4,0.6,0.8,1.0 \mathrm{mg} / \mathrm{ml})$ was added into $10 \mathrm{ml}$ chitosan solution, respectively. The drug rosiglitazone maleate was dissolved in the chitosan solution. Tween-80 (\%) solution was added to get a final suspension of chitosan nanoparticles. The suspension was stirred for $1 \mathrm{~h}$ using a magnetic stirrer. Three kinds of phenomena were observed: solution, aggregates and opalescent suspension. The zone of opalescent suspension was further examined as nanoparticles.

\section{Physicochemical characterization of nanoparticles}

\section{Fourier-transform Infrared Spectroscopy}

Fourier-transform infrared (FTIR) spectra of the chitosan and cross-linked chitosan were obtained from 8201 PC, Schimadzu (Tokyo, Japan). The pellets were prepared on a $\mathrm{KBr}$ press. The spectra were scanned over the wave number range of 4600 to $400 \mathrm{~cm}^{-1}$. The pellet was placed in the light path and the spectrum was obtained.

\section{Particle morphology}

Particle morphology was analyzed by a transmission electron microscope (TEM, Margagni-268-D, FEI,
Netherlands) using an acceleration voltage of $200 \mathrm{kV}$. Specimens were prepared by dropping the sample solution on to an upper grid, and then a drop of $2 \%$ uranyl acetate was added to give negative stain. The grid was then allowed to stand for $1 \mathrm{~min}$ and excess staining solution was removed by draining. The specimens were air dried and examined using TEM. ${ }^{12,13}$

\section{Particle size analysis}

Particle size of the nanoparticles was determined using a Zeta sizer 3000 HS (Malvern Instruments, Malvern, UK). Samples were diluted with phosphate buffer saline $\mathrm{pH} 7.4$ and the measurements were performed at a scattering angle of 90 - and at a temperature of $25^{\circ} \mathrm{C}$. The diameter is calculated from the autocorrelation function of the intensity of light scattered from particles, assuming a spherical form for the particles. The polydispersity index $(\mathrm{Pdl})$ is a measure of dispersion homogeneity and ranges from 0 to 1 . Values close to 0 indicate a homogeneous dispersion while those greater than 0.3 indicate high heterogeneity or broad size distribution. ${ }^{14}$

\section{Zeta potential}

The particle charge was quantified measuring the zeta potential using a Zeta sizer $3000 \mathrm{HS}$ (Malvern Instruments, Malvern, UK). Samples were diluted with phosphate buffer saline $\mathrm{pH}$ 7.4. For the measurements samples were placed in the electrophoretic cell, where a potential of $150 \mathrm{mV}$ was established. The zeta potential value was calculated by the software using Smoluchowski's equation. The results are presented as the mean of 3 determinations \pm S.D. ${ }^{14}$

\section{Entrapment efficiency}

Each suspension was centrifuged at $15,000 \mathrm{~g}$ for $40 \mathrm{~min}$ at $2^{\circ}$ to $4{ }^{\circ} \mathrm{C}$ to separate the free drug in the supernatant from the drug incorporated in the nanoparticles. Concentration of Rosiglitazone maleate was determined by visible spectrometry at $243.5 \mathrm{~nm}$. The amount of the drug incorporated in nanoparticles was calculated from the difference in drug concentrations between the supernatant and the original given concentrations. ${ }^{15}$ The entrapment efficiency was calculated according to the following equation:

\section{The entrapment efficiency $(\%)=\underline{\text { Weight of the total drug }- \text { weight of free drug }} \times 100$ \\ Weight of total drug}

\section{Effect of surfactant on the drug loading capacity}

The effect of surfactant on drug loading capacity was determined by adding various concentrations of surfactant. The batch of nanoparticles with minimum drug loading was selected for this study. After the addition of surfactant in the Chitosan solution, the other steps were followed as it is described in the preparation of the drug loaded nanoparticles. Drug loading capacity of the batch without addition surfactant was checked and it was then compared with the percentage drug loading after addition of surfactant. ${ }^{16}$

\section{In vitro release studies of drug loaded batches by dialysis membrane}

In-vitro release of all batches was carried out by employing a diffusion cell (float a lyzer). A quantity of nanoparticles suspension which is equivalent to $4 \mathrm{mg}$ of drug was taken in a Himedia dialysis membrane (cut off $14 \mathrm{KDa}$ ) fixed to one end of the apparatus to result a permeation cell. The nanoparticles were taken in the cell and the cell was immersed in a beaker containing $50 \mathrm{ml}$ of phosphate buffer $(\mathrm{pH} 7.4)$ as receptor compartment. The cell was immersed to a depth of $1 \mathrm{~cm}$ below the surface of the receptor 
solvent. The medium in the receptor compartment was agitated continuously using a magnetic stirrer and a temperature of $35 \pm 2 \circ \mathrm{C}$ was maintained within the diffusion chambers. $1 \mathrm{ml}$ of the sample from receptor compartment was taken at various prefixed intervals of time period of over $24 \mathrm{~h}$ and each time fresh buffer was reintroduced in the receiver chamber. The withdrawn sample was estimated spectrophotometrically at 243.5 $\mathrm{nm} .{ }^{15,17}$

\section{Evaluation of in vitro release kinetics}

In order to investigate the mechanism of release, the data were analyzed with the following mathematical models: zero order kinetic (Eq. 1), first order kinetic (Eq. 2) and Higuchi kinetic (Eq. 3).

$\mathrm{Qt}=\mathrm{Kot}$

$\ln Q t=\ln Q 0-K 1 t$

$\mathrm{Qt}=\mathrm{Kht} 1 / 2$

The following plots were made: Qt vs. $t$

(Zero order kinetic model),

In (Q0 -Qt) vs. $t$ (first order kinetic model) and $Q t$ vs. $t 1 / 2$ (Higuchi model), where $Q$ t is the percentage of drug released at time $\mathrm{t}, \mathrm{Q} 0$ is the initial amount of drug present in the formulation and $\mathrm{KO}, \mathrm{K} 1$ and $\mathrm{Kh}$ are the constants of the equations. Further, to confirm the

mechanism of drug release, the first $60 \%$ of drug release was fitted in Korsmeyer-Peppas model (Eq. 4)

$\mathrm{Mt} / \mathrm{M}_{\mathbf{c}}=\mathrm{Kp} \mathrm{tn}$

Where

$\mathrm{Mt} / \mathrm{M}=$ are the fraction of the drug release at time $t, \mathrm{Kp}$ is the rate constant and " $n$ " is the release exponent. The value of " $n$ " is used to characterized if ferent release mechanisms and is calculated from the slope of the plot of log of fraction of drug released (Mt/M ) vs. log of time. ${ }^{18}$

\section{Stability}

The nanoparticles formulations were stored for stability profile for 1 month in different environment conditions such as $2^{\circ}-8^{\circ} \mathrm{C}$, room temperature and $45^{\circ} \mathrm{C}$. The stability of drug loaded nanoparticles was evaluated every week in terms of its drug leakage into the storage medium buffer saline $\mathrm{pH} 7.4 .^{14,19}$

\section{RESULTS AND DISCUSSION}

\section{Development of polymeric nanoparticles}

The polymeric nanoparticles were prepared by ionic gelation method with four different ratios of polymer. This method is comparatively easy to prepare than the other techniques. This mild technique involves the mixing of two aqueous solutions at ambient temperature without sonication or using organic solvents. ${ }^{20}$ Various formulation were made with different initial concentrations of chitosan (1-6 $\mathrm{mg} / \mathrm{ml}$ ) and sodium tripolyphosphate solutions (1-5 $\mathrm{mg} / \mathrm{ml}$ ) to establish preparation conditions at which were formed and more over $3 \mathrm{mg} / \mathrm{ml}$ chitosan concentration and $4.6 \mathrm{mg} / \mathrm{ml}$ sodium tripolyphosphate was choosen for the study, as reported in literature. ${ }^{11}$

Chitosan was dissolved in acetic aqueous solution, added TPP under magnetic stirring at room temperature. The drug and Tween- 80 solution was added to get a final suspension of chitosan nanoparticles. The polymeric nanoparticles were prepared in four different ratios of polymer viz. 1:1, 1:2, 1:3 and 1:4. However, based on the nanoparticle recovery and drug entrapment efficiency, among the four different ratios, 1:3 ratio is selected as the best ratio than the other three. The other three ratios produced low drug entrapment which causes high drug wastage during the preparation procedure itself and showed low nanoparticle recovery, and poor yield. These have been repeatedly tried for three times, for reproducibility and for consistency.

\section{Physicochemical characterization of Nanoparticles}

Prepared nanoparticles were characterized for their FTIR, particle size, surface properties, morphology, entrapment efficiency, stability and in vivo release study.

\section{FTIR spectra of chitosan, rosiglitazone maleate and rosiglitazone-loaded nanoparticles}

A characteristic band at $3449 \mathrm{~cm}^{-1}$ is attributed to $-\mathrm{NH} 2$ and $-\mathrm{OH}$ groups stretching vibration and the band for amide $I$ at $1655 \mathrm{~cm}^{-1}$ is seen in the infrared spectrum of chitosan (Fig. 1A). In chitosan-TPP nanoparticles, the 1655 $\mathrm{cm}^{-1}$ peak of $-\mathrm{NH} 2$ bending vibration shifts to $1554 \mathrm{~cm}^{-1}$ and a new sharp peak $1645 \mathrm{~cm}^{-1}$ appears. The disappearance of the band could be attributed to the linkage between the phosphoric and ammonium ions. The cross-linked chitosan also showed a peak for $\mathrm{P}=\mathrm{O}$ at 1155 $\mathrm{cm}^{-1}$. Many researcher also observed similar results in their study of formation of chitosan nanoparticles and chitosan film treated with phosphate. ${ }^{21,22,23}$ So we suppose that the tripolyphosphoric groups of TPP were linked with ammonium groups of chitosan in nanoparticles. Compared with the spectrum of rosiglitazone maleate (Fig. 1B), in the spectrum of rosiglitazone maleate -loaded nanoparticles (Fig. 1C), the absorption peak of $1718 \mathrm{~cm}^{-1}$ (carboxyl group absorption peak) disappears and a new shoulder peak $1453 \mathrm{~cm}^{-1}$ (salt of carboxyl) appears. The results indicate that the presence of the electrostatic interactions between carboxyl groups of rosiglitazone maleate and amino groups of chitosan.

\section{Morphology of nanoparticles}

Particle morphology was analyzed using TEM using an acceleration voltage of $120 \mathrm{kV}$. Results showed that the surface of nanoparticles were uniform and of definite shape. The particles morphology was showed in Fig 2. 


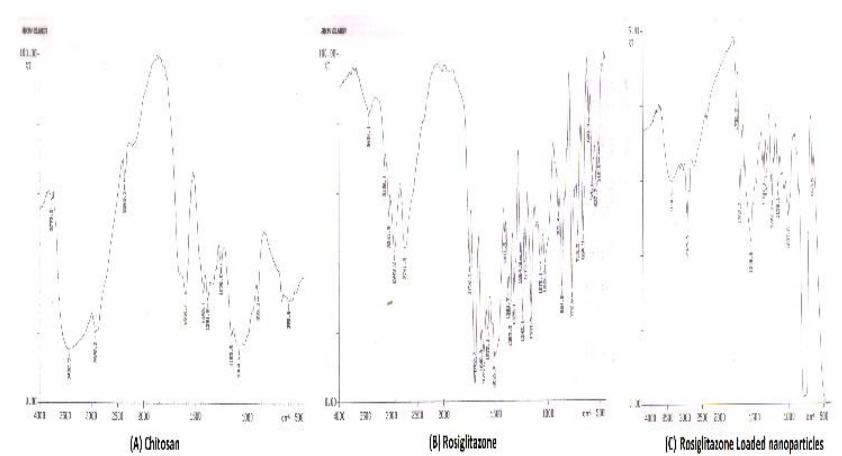

Figure 1: FTIR spectra of: (A) chitosan, (B) Rosiglitazone and (C) Rosiglitazone loaded nanoparticles.

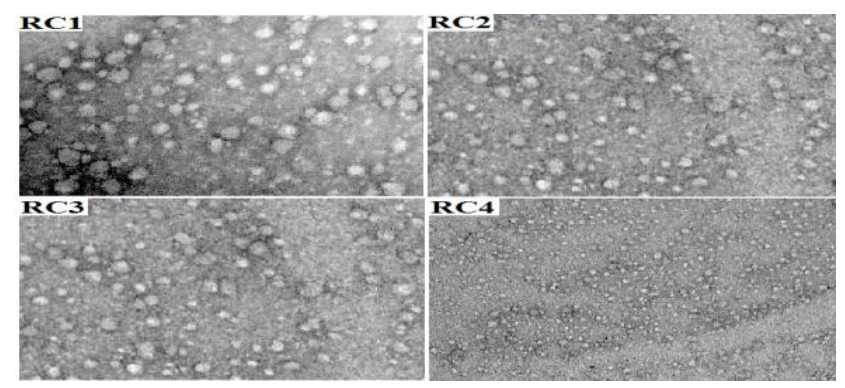

Figure 2: TEM of Rosiglitazone loaded chitosan nanoparticles (RC1 RC2 RC3 and RC4)

\section{Zeta size}

Particle size is often used to characterize nanoparticles, because it facilitates the understanding of the dispersion and aggregation. ${ }^{24}$ Nanoparticles produced were of submicron size and had low poly dispersity which indicates relatively narrow particle size distribution for Rosiglitazone loaded chitosan preparations. The mean diameter and poly dispersity index (PDI) of polymeric nanoparticles was found to be $1120 \mathrm{~nm}$ and $0.858 \mathrm{PDI}$. The raw data was correlated to $Z$ average means size by cumulative analysis. Z-average (d.nm) of chitosan nanoparticles (RC3) was found 1120, shown in Fig 3.

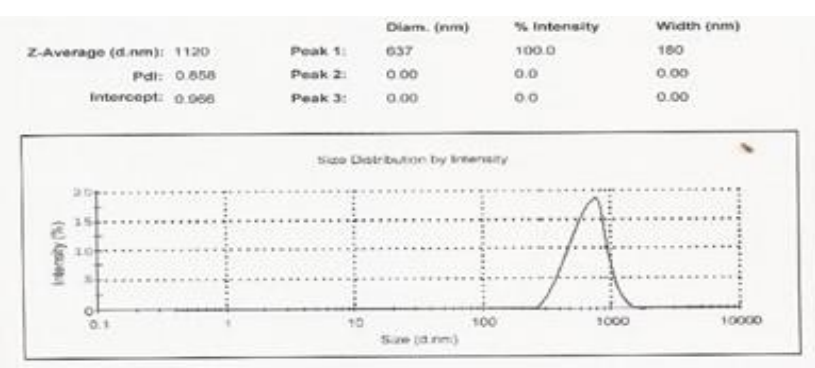

Figure 3: Zeta size of Rosiglitazone loaded chitosan nanoparticles of $1: 3$ ratio

\section{Zeta potential}

Zeta potential is used to characterize the surface properties of nanoparticles and colloidal drug delivery system. Zeta-potential of chitosan nanoparticles (RC3) was found $28.0 \mathrm{mV}$, shown in Fig 4.

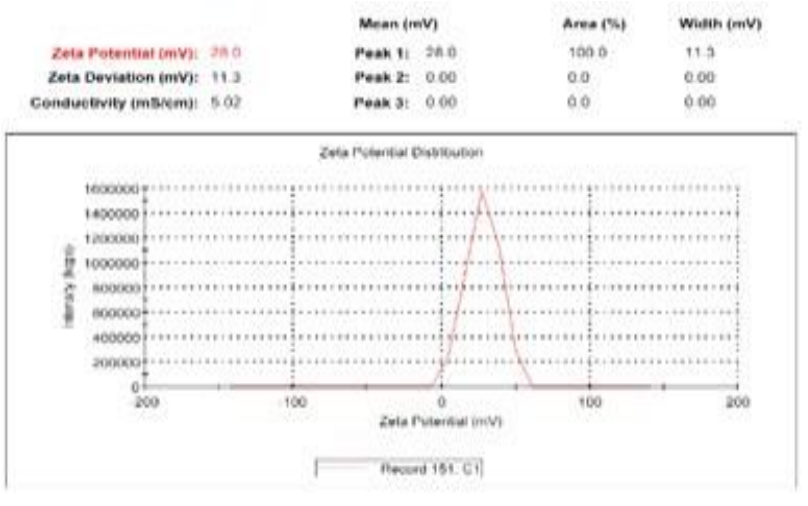

Figure 4: Zeta potential of Rosiglitazone loaded chitosan nanoparticles (RC3)

\section{Entrapment efficiency in nanoparticles}

The entrapment efficiency of chitosan nanoparticles loaded with drug was found for different ratio of polymer showed in Table 1 . The study was focused on the encapsulation efficiency of drug by cross linking method using sodium triphosphate as cross linking agents. On the basis of in vitro release studies it was found that a bettersustained and prolonged release was obtained in case of STPP and encapsulation efficiency of the RC3 was found to be $90.61 \%$. It was assumed that the intensive ionic interaction between the polymer and the drug may lead to an increase in entrapment of the drug in nanoparticles.

Table 1: Entrapment efficiency of chitosan nanoparticles

\begin{tabular}{cccc}
\hline SN & Formulation Code & Drug Polymer ratio & \% Entrapments efficiency \\
\hline 1 & RC1 & $1: 1$ & $91.41 \pm 0.37$ \\
2 & RC2 & $1: 2$ & $89.06 \pm 1.26$ \\
3 & RC3 & $1: 3$ & $90.61 \pm 0.27$ \\
4 & RC4 & $1: 4$ & $88.78 \pm 0.26$ \\
\hline
\end{tabular}

\section{Effect of Chitosan/TPP concentration on the encapsulation of rosiglitazone maleate}

When TPP concentration was $1 \mathrm{mg} / \mathrm{ml}$, too high chitosan concentration $(4 \mathrm{mg} / \mathrm{ml})$ made encapsulation extremely difficult and too low chitosan concentration $(0.5 \mathrm{mg} / \mathrm{ml})$ made some aggregates with large diameter form. The formation of nanoparticles is only possible within some moderate concentrations of chitosan and TPP. As for gelation between TPP solution of $1 \mathrm{mg} / \mathrm{ml}$ and chitosan solution of $1-3 \mathrm{mg} / \mathrm{ml}$, we usually observed that some opalescent suspension was formed, which was further examined as nanoparticles. Table1 shows that increase in chitosan concentration led to decrease of encapsulation efficiency of rosiglitazone maleates.

It has been previously reported that the highly viscous nature of the gelation medium hinders the encapsulation of drug in the study of chitosan microspheres. ${ }^{25}$ So it was supposed that relatively lower viscosity of chitosan with lower concentration (such as $1-3 \mathrm{mg} / \mathrm{ml}$ ) and $4.6 \mathrm{mg} / \mathrm{ml}$ sodium tripolyphosphate promotes the encapsulation of rosiglitazone maleate and gelation between chitosan and TPP. 


\section{Effect of surfactant on drug loading capacity}

Because of larger surface area and attractive force between the particles, the chance of possible aggregation is high in small sized particles. To overcome such aggregations, which were not solicited, an addition of a surfactant in the preparation was necessary. Tween-80 appeared to be the most suitable surfactant in reducing aggregation between nanoparticles.

\section{In vitro release of rosiglitazone maleate from the nanoparticles}

Figure 5 displayed the release profile of rosiglitazone maleate from chitosan nanoparticles. It was apparent that rosiglitazone maleate release in vitro showed a very rapid initial burst, and then followed by a very slow drug release. Zhou reported about microspheres and revealed that the release involves two different mechanisms of drug molecules diffusion and polymer matrix degradation. ${ }^{26}$

The burst release of drug is associated with those drug molecules dispersing close to the microsphere surface, which easily diffuse in the initial incubation time. The hypothesis is also suitable for rosiglitazone maleate release from nanoparticles. Rosiglitazone maleate nanoparticles diffuse easily through the surface or the pore of nanoparticles in a short time because of its small size. Therefore, the rapid dissolution process suggests that the release medium penetrates into the particles due to the hydrophilic nature of chitosan and dissolves the entrapped rosiglitazone maleate. In addition, the nanoparticles with huge specific surface area can adsorb rosiglitazone maleate.

In vitro release profiles obtained for each formulation showed three phases compositions:

(1) A first initial burst release of $30 \%$, due to the drug desorbed from the particles surface,

(2) A plateau for the following $8 \mathrm{~h}$, resulting from the only diffusion of the drug dispersed in the polymer matrix,

(3) A constant sustained release of the drug, resulting from the diffusion through the polymer wall as well as its erosion, so the first burst release is also possibly due to the part of rosiglitazone maleate desorbed from nanoparticle surface.

However, the drug which was covalent bound to the nanoparticles continues to release in a sustained and prolonged manner and the total amount of drug release in terms of percentage entrapment was almost $67 \%$ from chitosan nanoparticles at the end of $32 \mathrm{~h}$. In order to determine the release model which best describes the pattern of drug release, the in vitro release data were substituted in zero order, first order and diffusioncontrolled release. The release pattern followed the Korsmeyer Peppas equation.

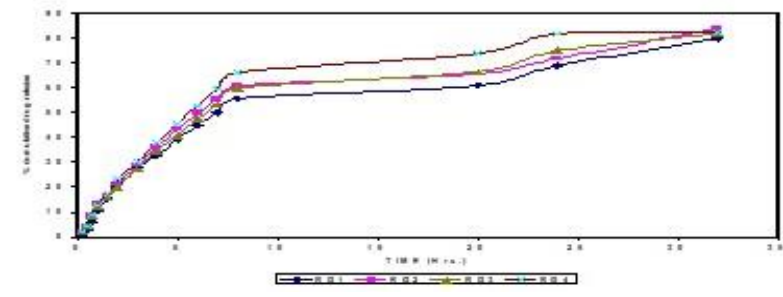

Figure 5: In vitro release profile of rosiglitazone loaded chitosan nanoparticles of 1:3 ratio

Stability

Nanoparticles made with chitosan were stored at $4^{\circ} \mathrm{C}$, room temperature and $45^{\circ} \mathrm{C}$ for one month. It could be deduced that no aggregation occurs during the storage and the formulations exhibit a good stability (Fig.6). After 3 months study, aggregation of nanoparticles was observed and nanoparticles were not found under nanometer size range (Tab. 2).

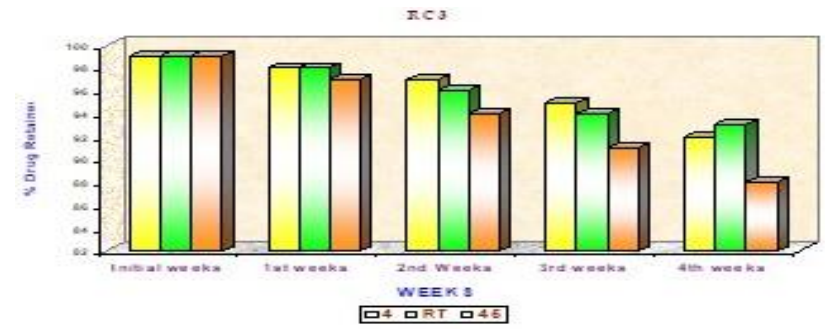

Figure 6: Stability study of rosiglitazone loaded chitosan nanoparticles of $1: 3$ ratio

Table 2: Particle size distribution initial and after 3 month

\begin{tabular}{ccll}
\hline \multicolumn{2}{c}{ In itial particle size } & \multicolumn{2}{l}{ Particle size after $\mathbf{3}$ months } \\
TEM (nm) & Zeta size $(\mathbf{n m})$ & TEM $(\mathbf{n m})$ & Zeta size $(\mathbf{n m})$ \\
\hline $86 \pm 18.2$ & 1120 & $110 \pm 19.6$ & \\
\hline
\end{tabular}

\section{CONCLUSION}

This work shows a systematic study on the chemical physical characterization of nanoparticles formed by chitosan chains electrostatically linked by ionic interactions. This kind of particles is of great interest for pharmaceutical applications. Rosiglitazone loaded chitosan nanoparticles were successfully formulated by ionic gelation method. Different investigations on preparation, characterization and in vitro release of nanoparticles were carried out and performance of the formulations was evaluated. The proposed rosiglitazone loaded chitosan nanoparticles illustrates an effective way, to prolong drug release. The developed nanoparticles are safer and are the need of the hour for pharmaceutical industry as an alternative drug delivery system for the treatment of highly prevalent and chronic disease like type Il diabetes mellitus. To optimize this drug delivery system, and for deeper understanding of different mechanisms, further studies are still required. Accordingly, the next step of this work has been planned to optimize various parameters which influence efficacy and bioavailability in vivo. 
Acknowledgements: Authors are thankful to Torrent Pharmaceutical Ltd, Ahemdabad, India for providing the gift sample of rosiglitazone maleate. Authors are also thankful to Dr S. K. Jain and Dr. A. K. Jain, Dr. H. S. Gour University for his valuable suggestions.

\section{REFERENCES}

1. Sunil KJ, Awasthi AM, Jain NK, Agarwal GP. Calcium silicate based micro- spheres of repaglinide for gastro retentive floating drug delivery: preparation and in vitro characterization. J. Control Release. 107, 2005, 300-309.

2. Soppimath KS, Kulkarni AR, Aminabhavi TM. Development of hollow microspheres as floating controlled-release systems for cardiovascular drugs: Preparation and release characteristics. Drug Dev. Ind. Pharm. 27, 2001, 507.

3. Govender T, Stolnik S, Martin CG, Illum L, Stanley SD. PLGA nanoparticles prepared by nanoprecipitation: drug loading and release studies of a water-soluble drug. J. Control Release. 57, 1999, 171-185.

4. Bala I, Hariharan S, Kumar MN. PLGA nanoparticles in drug delivery the state of art. Crit. Rev. Ther. Drug Carrier Syst. 21, 2004, 387- 482.

5. Scientific discussion EMEA, 2005.

6. Lam CW, James JT, McCluskey R, Hunter RL. Pulmonary toxicity of single-wall carbon nanotubes in mice, 7 and 90 days after intratracheal instillation. Toxicol. Sci. 77, 2004, 126-134.

7. Ho M, Chen Z, Xing G, Yuan H, Chen C, Zhao F, Zhang C, Zhao $Y$. Ultrahigh reactivity provokes nanotoxicity: explanation of oral toxicity of nano- copper particles. Toxicol. Lett. 175, 2007, 102-110.

8. Oberdorster E, Oberdorster J. Nanotoxicology: an emerging discipline evolving from studies of ultrafine particles. Environ. Health Perspect. 113, 2005, 823-839.

9. Wiesner MR, Lowry GV, Alvarez P, Dionysiou D, Biswas P. Assessing the risks of manufactured nanomaterials. ES\&T. 40, 2006, 4336-4345.

10. He P, Davis SS, Illum L. In vitro evaluation of the mucoadhesive properties of chitosan microspheres. Int. J. Pharm. 166, 1998, 75-88.

11. Bhumkar RD, Pokharkar BV. Studies on effect of $\mathrm{pH}$ on crosslinking of chitosan with sodium tripolyphosphate. AAPS Pharma Sci.Tech. 7(2), 2006, Article 50.

12. Benita $S$, Levy MY, Magenhein B, A new in vitro technique for the evaluation of drug release profile from colloidal carriersultrafiltration technique at low pressure. Int. J. Pharmaceutics. 94, 1993, 115-123.
13. Eerikainen H, Peltonen L, Raula J, Hirvonen J, Kauppinen EI, Nanoparticles containing ketoprofen and acrylic polymers prepared by an aerosol flow reactor method. AAPS Pharm Sci. Tech. 5(4), 2004, 68.

14. Pignatello R, Ricupero N, Bucolo, Maugeri F, Maltese A, Puglisi $G$, Preparation and characterization of eudragit retard nanasuspensions for the ocular delivery of cloricromene. AAPS Pharm Sci. Tech. 7(1), 2006, 27.

15. Bellare J, Banerjee R, Das S. Aspirin loaded albumin nanoparticles by coacervation: Implications in drug delivery. Trends Bio Artif Organs. 1(2), 2005, 8.

16. Kauppinen El. Nanoparticles containing ketoprofen and acrylic polymers prepared by an aerosol flow reactor method. AAPS Pharm Sci. Tech. 5(4), 2004, 68.

17. D'souza SS, Deluca PP. Development of a dialysis in vitro release method for biodegradable microspheres. AAPS Pharm Sci. Tech. 6(2), 2005, 42.

18. Costa P, Lobo JMS. Modelling and comparison of dissolution profiles. Euro. J. Pharm. Sci. 13, 200, 123-133.

19. Reddy LH, Murthy R. Etoposide-loaded nanoparticles made from glyceride lipids: Formation, characterization, in-vitro drug release, and stability evaluation. AAPS Pharma Sci. Tech. 6(2), 2005, E158-E616.

20. Van der Lubben IM, Verhoef JC, Borchard G, Junginger HE. Chitosan and its derivatives in mucosal drug and vaccine delivery. Eur. J. Pharm. Sci. 14, 2001, 201-207.

21. Xu Y, Du Y. Effect of molecular structure of chitosan on protein delivery properties of chitosan nanoparticles. Int. J. Pharm. 250, 2003, 215-226.

22. Knaul JZ, Hudson SM, Creber KAM. Improved mechanical properties of chitosan fibres. J. Appl. Polym. Sci. 72, 1999, 1721-1731.

23. Wang X, Ma J, Wang Y, He B. Structural characterization of phosphorylated chitosan and their applications as effective additives of calcium phosphate cements. Biomaterials. 22, 2001, 2247-2255.

24. Mourdikoudis S, Pallares RM, Thanh NTK. Characterization techniques for nanoparticles: comparison and complementarity upon studying nanoparticle properties. Nanoscale. 10, 2018, 12871-12934.

25. Vandenberg GW, Drolet C, Scott SL, No“ue JD. Factor affecting protein release from alginate-chitosan coacervate microcapsules during production and gastric/intestinal simulation. J. Control Release. 77, 2001, 297-307.

26. Zhou SB, Deng XM, Li XH. Investigation on a novel core-coated microspheres protein delivery system. J. Control Release. 75, 2001, 27-36.

Source of Support: None declared.

Conflict of Interest: None declared.

For any question relates to this article, please reach us at: editor@globalresearchonline.net

New manuscripts for publication can be submitted at: submit@globalresearchonline.net and submit_ijpsrr@rediffmail.com 